\title{
Variation at the Hepatic Lipase and Apolipoprotein Al/CIII/AIV Loci Is a Major Cause of Genetically Determined Variation in Plasma HDL Cholesterol Levels
}

\author{
Jonathan C. Cohen, ${ }^{*}$ Zifen Wang, ${ }^{*}$ Scott M. Grundy, ${ }^{\star \ddagger}$ Marcia Regier Stoesz, $\$$ and Rudy Guerra ${ }^{\S}$ \\ *The Center for Human Nutrition, ${ }^{\ddagger}$ Departments of Clinical Nutrition and Internal Medicine, University of Texas Southwestern Medical \\ Center, Dallas, Texas 75235-9052; and ${ }^{\S}$ The Department of Statistical Sciences, Southern Methodist University, \\ Dallas, Texas 75275-0332
}

\begin{abstract}
Genetic factors have been shown to play an important role in determining interindividual variation in plasma HDL-C levels, but the specific genetic determinants of HDL cholesterol (HDL-C) levels have not been elucidated. In this study, the effects of variation in the genomic regions encoding hepatic lipase, apolipoprotein AI/CIII/AIV, and the cholesteryl ester transfer protein on plasma HDL-C levels were examined in 73 normotriglyceridemic, Caucasian nuclear families. Genetic factors accounted for $56.5 \pm 13 \%$ of the interindividual variation in plasma HDL-C levels. For each candidate gene, adjusted plasma HDL-C levels of sibling pairs who shared zero, one, or two parental alleles identicalby-descent were compared using sibling-pair linkage analysis. Allelic variation in the genes encoding hepatic lipase and apolipoprotein AI/CIII/AIV accounted for 25 and $22 \%$, respectively, of the total interindividual variation in plasma HDL-C levels. In contrast, none of the variation in plasma HDL-C levels could be accounted for by allelic variation in the cholesteryl ester transfer protein. These findings indicate that a major fraction of the genetically determined variation in plasma HDL-C levels is conferred by allelic variation at the hepatic lipase and the apolipoprotein AI/CII/AIV gene loci. (J. Clin. Invest. 1994. 94:2377-2384.) Key words: lipoproteins HDL • lipoproteins HDL cholesterol • hepatic lipase - apolipoprotein AI - cholesteryl ester transfer protein
\end{abstract}

\section{Introduction}

Epidemiologic studies have provided strong evidence for an inverse relationship between plasma high density lipoprotein cholesterol (HDL-C $)^{1}$ levels and coronary heart disease (CHD) (1). Population-based studies have revealed that low HDL-C levels are a powerful risk factor for CHD (2-4), and several clinical trials have noted that increasing plasma levels of HDL$\mathrm{C}$ are correlated with a reduction in $\mathrm{CHD}$ risk $(5,6)$. These

Address correspondence to Jonathan C. Cohen, Ph.D., Center for Human Nutrition, UT Southwestern Medical Center, 5323 Harry Hines Blvd, Dallas, TX 75235-4837. 1994.

Received for publication 27 May 1994 and in revised form 15 August

1. Abbreviations used in this paper: CETP, cholesteryl ester transfer protein; CHD, coronary heart disease; HDL-C, HDL cholesterol.

J. Clin. Invest.

(C) The American Society for Clinical Investigation, Inc.

$0021-9738 / 94 / 12 / 2377 / 08 \quad \$ 2.00$

Volume 94, December 1994, 2377-2384 findings have stimulated extensive investigation into the determinants of plasma HDL-C levels.

Plasma HDL-C levels are clearly modulated by environmental variables such as obesity $(7,8)$, exercise $(9)$, and smoking $(10,11)$. Genetic factors also have a significant role. Data from family and twin studies indicate that genetic variation probably accounts for between 40 and $60 \%$ of the total variation in plasma HDL-C levels $(12,13)$. Since plasma HDL-C levels do not segregate as a mendelian trait in most families, the specific genes that determine plasma HDL-C levels cannot be identified using classical methods of segregation analysis. Analyses of the genetic architecture of plasma HDL-C levels have therefore used biometrical methods for which genotype data are not required (14). Evidence for major gene effects on HDL-C levels have been found in some of these studies (15), but not in others $(14,16)$. Therefore it is not clear whether a single gene (major gene), a few genes (oligogenes), or many genes (polygenes) account for the interindividual variation of plasma HDL-C levels. Other investigators have performed association studies to test for linkage disequilibrium between HDL-C levels and various DNA polymorphisms (17). Several associations between plasma HDL-C levels and DNA polymorphisms in genes involved in HDL metabolism have been reported $(18,19)$, but the associations observed have generally been weak, and often inconsistent from one study to another. Accordingly, elucidation of the specific genetic determinants of plasma HDL-C levels in humans has been very limited.

A promising approach to the genetic analysis of quantitative traits was developed by Haseman and Elston (20). The method uses data from sibling pairs to determine the effect of variation in a candidate gene on interindividual variation in a trait. If allelic variation in a candidate gene is associated with variation in a trait, then trait levels should be more similar in siblings sharing two identical alleles of the candidate gene than in siblings sharing neither allele. Conversely, if variation in a candidate gene does not influence a trait, then concordance for trait levels among siblings should be independent of the number of alleles of the candidate gene they share. This approach offers two major advantages. First, no a priori assumptions are made concerning the mode of inheritance of the trait, therefore information regarding allele frequencies or the phenotypes associated with specific alleles of the candidate gene is not required. Second, the segregation of candidate genes in a nuclear family can usually be unambiguously determined using polymorphisms in or near the candidate gene. Since the fidelity of DNA transmission from parents to their offspring is very high, genotype data can be used to define alleles by descent. By using genetic markers in or near any given candidate gene, the proportion of variance of any trait that is attributable to sequence variation in the candidate gene can be inferred. Boerwinkle et al. (21) were the first to apply this method to a trait for which a fully informative 
genetic marker was available. They used the highly informative length polymorphism at the apolipoprotein(a) locus to distinguish parental alleles in 40 nuclear families, and found that allelic variation in or near the apolipoprotein (a) gene accounted for $90 \%$ of the variation in plasma lipoprotein(a) levels (21).

The Haseman-Elston procedure has been used to investigate the genetic determinants of HDL-C in a large pedigree with excess coronary heart disease, but the results were limited by the low information content of polymorphisms at the candidate loci (22). In this report we have used the Haseman-Elston sibling-pair method to examine the effects of genetic variation at three candidate loci, those encoding hepatic lipase, apolipoprotein $\mathrm{AI} / \mathrm{CIII} / \mathrm{AIV}$, and the cholesteryl ester transfer protein (CETP), on variation in plasma HDL-C levels. These genes were considered to be good candidates because mutations that abolish their function cause significant changes in plasma HDLC levels (23-25), and normal physiological variation in hepatic lipase activity (26) and plasma apolipoprotein AI levels (27) are strongly correlated with plasma concentrations of HDL-C. To control for spurious correlations between HDL-C values and candidate genes we also determined the contribution of a physiologically unrelated gene, apolipoprotein(a). Our findings indicate that a major fraction of the variation in the plasma HDL-C levels of normotriglyceridemic individuals is determined by allelic variation in the genes encoding hepatic lipase and apolipoprotein AI/CIII/AIV.

\section{Methods}

\section{Subjects}

The study protocol was approved by the Institutional Review Board of the University of Texas Southwestern Medical Center at Dallas. Caucasian American families in which both parents and three or more children were available for sampling were recruited by advertisements in newspapers, churches, and health centers. Fasting blood samples were drawn into $10 \mathrm{ml}$ vacuum tubes containing sodium EDTA. Plasma was separated by centrifugation and stored at $4^{\circ} \mathrm{C}$ until analysis. Genomic DNA was isolated from the white blood cell pellet using an automated DNA extractor (Applied Biosystems, Foster City, CA). All available family members were sampled, but individuals who were taking lipid-lowering drugs or hormones were excluded from the analyses. Since high plasma triglyceride concentrations are strongly associated with low HDL-C levels, individuals with plasma triglyceride concentrations $>200 \mathrm{mg} /$ dl were excluded from the analysis.

\section{Assay of plasma lipids}

Plasma cholesterol and triglyceride concentrations were determined in duplicate by enzymatic assay using commercial reagents (Cholesterol/ HP; Boehringer Mannheim Biochemicals, Indianapolis, IN, and Triglycerides GPO-TRINDER; Sigma Chemical Co., St. Louis, MO). HDL-C was measured in the supernatant after precipitation of apolipoprotein B-containing lipoproteins with sodium phosphotungstate (28). Samples from all individuals in a family were analyzed in the same assay. Intraassay variation was $<3 \%$ for plasma cholesterol and triglyceride, and $<5 \%$ for plasma HDL-C.

\section{Analysis of the hepatic lipase, apolipoprotein AI/CIII/ $A I V$, and CETP genes}

For each candidate gene, the four parental alleles of each nuclear family were distinguished using single-strand conformation polymorphisms and microsatellite polymorphisms published previously (29-38). Microsatellite polymorphisms located within 5 centiMorgans of the candidate genes were identified from high resolution linkage maps of the human genome $(31,33)$. In addition, we identified three new single-strand
Table I. Oligonucleotides Used to Amplify Single-strand Conformation Polymorphisms (SSCPs) Identified in the Apolipoprotein AI and CETP Genes

\begin{tabular}{llll}
\hline & $\begin{array}{c}\text { Oligo- } \\
\text { Location }\end{array}$ & \multicolumn{4}{l}{ Oligonucleotide Sequence } \\
\hline Apo AI & A7 & 5' CTG AAG CCA CCC CAC TCA GCC AGG C 3' \\
Intron 2 & A10 & 5' GGA CAC ATA GTC TCT GCC GCT GTC T 3' \\
CETP & CE17-5' & 5' CTA TCT GGC TCT GAC ACT TGA TGA T 3' \\
Intron 7 & CE17-3' & 5' GAG AGT ACA GTA CAT GGA TGA GTG C 3' \\
CETP & CE18-5' & 5' GTC ATC CTC TAC AGC AGT GGA TTG T 3' \\
Intron 8 & CE18-3' & 5' GCA GTC CAT TGA CCA TCG GCT CCT G 3'
\end{tabular}

Amplifications were performed using a melting temperature of $94^{\circ} \mathrm{C}$ and an annealing/extension temperature of $68^{\circ} \mathrm{C}$. Reactions were performed in $10 \mu \mathrm{l} \mathrm{con-}$ taining $50 \mathrm{mM} \mathrm{KCl}, 10 \mathrm{mM}$ Tris (pH 8.3), $1.5 \mathrm{mM} \mathrm{MgCl}_{2}, 75 \mathrm{ng}$ of each primer, $100 \mu \mathrm{M}$ dNTP, $1 \mathrm{U}$ Taq DNA polymerase, and $1 \mathrm{pmol}\left[{ }^{32} \mathrm{P}\right] \mathrm{dCTP}(3,000 \mathrm{mCi} /$ mmol). Aliquots $(2 \mu \mathrm{l})$ from reactions containing CETP intron 7 were diluted in formamide dye ( $98 \%$ formamide, $0.1 \%$ bromophenol blue, $0.1 \%$ xylene cyanol). Reactions containing apolipoprotein AI intron 2 and CETP intron 8 were digested with PvuII and HaeIII, respectively, before dilution in formamide. The diluted samples were boiled for $1 \mathrm{~min}$, and then subjected to electrophoresis at $400 \mathrm{~W}$ on $6 \%$ polyacrylamide gels.

conformation polymorphisms (Table I). These are located in intron two of the apolipoprotein AI gene, and in introns seven and eight of the CETP gene. The segregation of all four parental alleles of each candidate gene was established unambiguously in each family using two or more informative polymorphisms. For each allele, at least one informative polymorphism was identified within the gene, thereby preventing misclassification of alleles due to recombination between the polymorphism and the candidate gene.

\section{Analysis of the apolipoprotein(a) gene}

Apolipoprotein(a) alleles were identified using either pulsed-field gel electrophoresis and Southern blotting (39) or single-strand conformation polymorphisms (40), as described in detail previously.

\section{Statistical methods}

(a) Data adjustment. Plasma HDL-C levels vary with age and sex. To include data from men and women of different ages in the analysis, plasma HDL-C levels were adjusted for the effects of age and sex by transforming each observation $y$ to a z-score; $z=(y-\mu) / \sigma$ where $\mu$ and $\sigma$ are sex- and age-specific population parameters taken from Heiss et al. (41). To determine whether the adjustment procedure led to spurious results, additional analyses were performed after regression adjustment of HDL-C for age and sex. Plasma HDL-C levels were inversely related to plasma triglyceride levels, therefore the data were also analyzed after regression adjustment of HDL-C for age, sex, and triglyceride levels.

(b) Heritability estimation. The heritability index of plasma HDL$\mathrm{C}$ levels in our sample was estimated by regressing the average of the offspring plasma HDL-C values on the mid-parent values using weighted least squares. Weights to adjust for unequal family sizes were calculated as suggested by Falconer (42), with an unweighted least squares estimate used as the initial estimate of heritability in the weights.

(c) Estimation of variance components. The observed variation in plasma HDL-C levels that could be accounted for by each candidate gene was estimated using a sib-pair regression procedure developed by Haseman and Elston (20) and recently discussed by Boerwinkle et al. (21). Briefly, the trait value $x$ of an individual is modeled as an additive combination of genetic and environmental effects

$x=\mu+g+e$

where $\mu$ is an overall mean, $g$ represents the effect due to a given candidate gene, and $e$ denotes the residual environmental and additional 
polygenic factors that influence the trait. If $x_{i}(i=1,2)$ denotes the trait value of the $i^{h}$ sib in a sib pair, the procedure regresses the squared sibpair differences $\left(x_{1}-x_{2}\right)^{2}$ on the proportion $\pi$ of trait alleles the sib pair shares identical-by-descent. The expectation, conditional on $\pi$, of $\left(x_{1}-x_{2}\right)^{2}$ is

$E\left[\left(x_{i}-x_{2}\right)^{2}\right]=\alpha+\beta \pi$

with $\alpha$ and $\beta$ satisfying the equations

$\alpha=\sigma_{\delta}^{2}+2 \sigma_{g}^{2}$

$\beta=-2 \sigma_{g}^{2}$

where $\sigma_{g}^{2}$ is the variance attributable to polymorphism at the candidate gene locus $g$ and $\sigma_{\delta}^{2}$ is the variance attributable to sib-pair residual differences, i.e., $\sigma_{\delta}^{2}=\operatorname{var}\left(e_{1}-e_{2}\right)$. Assuming a common variance $\sigma_{e}^{2}$ for the individual residual effects $e_{i}, \sigma_{\delta}^{2}=2 \sigma_{e}^{2}-2 \operatorname{cov}\left(e_{1}, e_{2}\right)$.

The covariance term in this equation includes sibling covariance due to additional trait loci, polygenes, and shared environment. Correlations between siblings indicate that these factors did not contribute an appreciable fraction of the variance in HDL-C this sample. Therefore the covariance term in $\sigma_{\delta}^{2}$ was set to 0 in this analysis. Under these assumptions it follows that

$\sigma_{\delta}^{2}=2 \sigma_{e}^{2}$

The contribution by $g$ to the interindividual variation in the quantitative trait $x$ is denoted by $h_{g}^{2}$ and is defined as

$h_{g}^{2}=\sigma_{g}^{2} /\left(\sigma_{g}^{2}+\sigma_{e}^{2}\right)$

Estimates of the variance components $\sigma_{g}^{2}$ and $\sigma_{e}^{2}$ are obtained by fitting the regression model (2) by least squares and solving for these components in the system of equations given in (3) and (4).

An approximate standard error for the estimated variance contribution for each candidate gene was calculated by the method of statistical differentials, usually referred to as the delta method (43). This calculation requires the covariance matrix of the regression parameter estimates and an approximating standard error formula for the ratio of two parameter estimates.

Least squares was used to fit regression model (2). Since least squares estimates are very sensitive to outliers, such points were identified, removed, and the variance contribution was recalculated for the remaining data. Potentially outlying points were identified as those pairs $\left(x_{1}, x_{2}\right)$ which significantly influenced the parameter estimates as determined by DFBETA residual diagnostics (44). The adjusted HDL-C values of the individuals $x_{1}$ and $x_{2}$ were then compared to all siblings within their sibship. An individual $x_{i}$ was identified as an outlier if his/ her trait value differed markedly from the other siblings. All pairs $\left(x_{1}\right.$, $x_{2}$ ) involving an individual identified as an outlier were removed.

(d) Intraclass correlations. The correlation between siblings sharing both hepatic lipase alleles and both apolipoprotein AI/CIII/AIV alleles

Table II. Distribution of Sibship Sizes

\begin{tabular}{cc}
\hline Sibship size & No. of families \\
\hline 2 & 2 \\
3 & 20 \\
4 & 34 \\
5 & 12 \\
6 & 4 \\
7 & 1 \\
$9 *$ & 1
\end{tabular}

* This family had type III hyperlipidemia and was excluded from all analyses.

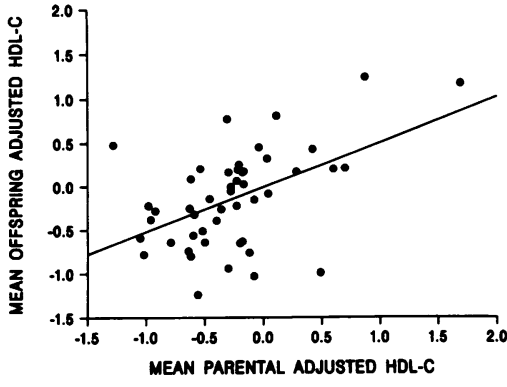

Figure 1. Scatter plot of mid-parent versus mean offspring adjusted plasma HDL-C levels. identical by descent, and between siblings sharing no alleles of either gene, were calculated using the intraclass correlation method (42). This method was used to avoid spurious correlations arising due to arbitrary ordering of the sib values (for example, by age or by adjusted HDL-C value).

\section{Results}

Genomic DNA and plasma samples for lipid and HDL-C levels were obtained from 459 individuals comprising 74 nuclear families. The distribution of sibship sizes is given in Table II. One family with type III hyperlipidemia, seven individuals who used medication known to influence HDL-C levels, and two individuals with possible monogenic hyperalphalipoproteinemia (adjusted HDL-C $>2.5$ SD above the mean) were excluded a priori from the analysis. 28 parents and 13 offspring with plasma triglyceride concentrations $>200 \mathrm{mg} / \mathrm{dl}$ were also excluded. The heritability of plasma HDL-C levels was calculated by regression of mean offspring adjusted HDL-C on mean parental adjusted HDL-C. Only those families $(n=48)$ in which both parents had triglyceride levels $<200 \mathrm{mg} / \mathrm{dl}$ were included in this calculation. In these families heritability of HDL-C was $56.5 \%$ with a standard error of $13 \%$ (Fig. 1 )

The four parental alleles of the genes encoding hepatic lipase, apolipoprotein AI/CIII/AIV, and CETP were unambiguously resolved in 71, 71, and 69 families, respectively (Table III). Sibling-pair differences in plasma HDL-C levels clearly

Table III. Proportion of Variation in Adjusted Plasma HDL-C Levels due to Allelic Variation in the Genes Encoding Hepatic Lipase, Apolipoprotein AI/CIII/AIV, CETP, and Apolipoprotein(a)

\begin{tabular}{lcccc}
\hline \multicolumn{1}{c}{ Gene } & No. of familes & $\hat{h}_{s}^{2}(z)$ & $\hat{h}_{g}^{2}(r)$ & $\hat{h}_{g}^{2}(o)$ \\
\hline Hepatic lipase & 71 & $25(381)$ & $23(381)$ & $21(370)$ \\
Apo AI/CIII/AIV & 71 & $22(382)$ & $20(382)$ & $16(371)$ \\
CETP & 69 & $0(371)$ & $0(371)$ & $0(360)$ \\
Apolipoprotein(a) & 48 & $0(286)$ & - & - \\
\hline
\end{tabular}

$\hat{h}_{g}^{2}$ is the proportion of total variance in adjusted plasma HDL-C that can be accounted for by allelic variation in the candidate gene. The number of sibling pairs used in each analysis is given in parentheses after $\hat{h}_{g}^{2}$. Individuals with possible monogenic hyperalphalipoproteinemia $(n=2)$, with plasma triglyceride levels $>200 \mathrm{mg} / \mathrm{dL}(n=13)$, and those who used lipid lowering medication $(n=7)$, were excluded from the analysis. The letter in parentheses indicates the adjustment procedure: $z$ indicates estimates based on z-score adjusted-HDL-C values, $r$ indicates estimates based on regression adjusted HDL-C levels, $o$ indicates estimates based on regression adjusted HDL-C values after exclusion of influential individuals. 


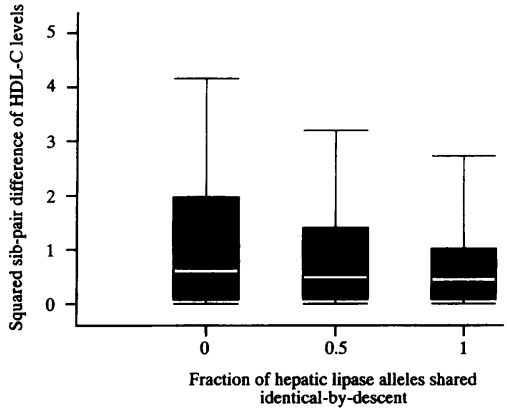

Figure 2. Distributions of squared sibling-pair differences of adjusted plasma HDL-C levels in siblings sharing none, one, or two hepatic lipase alleles identical-by-descent. Median values are indicated by white bars within boxes; upper and lower quartiles are indicated by the tops and bottoms of the boxes, re-

spectively; whiskers indicate the data points closest to three times the semi-interquartile range (one half the interquartile range) from the tops or bottoms of the boxes.

decreased with increasing proportion of alleles shared identicalby-descent for hepatic lipase (Fig. 2) and apolipoprotein AI/ CIII/AIV (Fig. 3), and regression analysis based on all pairs indicated that allelic variation in the genomic regions encoding hepatic lipase and apolipoprotein AI/CIII/AIV accounted for a substantial fraction of the variation in plasma HDL-C levels (Table III). In contrast, the sibling-pair differences in plasma HDL-C levels did not decrease with increasing proportion of CETP (Fig. 4) or apolipoprotein(a) alleles (Fig. 5) shared identical-by-descent. None of the variance in plasma HDL-C levels was attributable to allelic variation in these genes (Table III).

Several additional analyses were conducted to investigate the possibility of spurious results due to statistical artifact. Plasma HDL-C levels were adjusted for the effects of age and sex by a z-score transformation before analysis. To determine the effects of the adjustment procedure, the data were also analyzed after adjustment of HDL-C levels for the effects of age and sex using multiple linear regression, rather than the $\mathrm{z}$-score method. The variance estimates calculated for regression adjusted HDL-C values were very similar to those calculated for $\mathrm{z}$-score-adjusted HDL-C values (Table III), indicating that the results were not an artifact of the specific adjustment procedure.

The least squares estimates used in the Haseman-Elston procedure are sensitive to outliers. Points that may have significantly influenced the regression parameter estimates were identified using the DFBETA method. The data were then reanalyzed after exclusion of 11 individuals found to influence the hepatic lipase and apolipoprotein AI/CIII/AIV estimates, and 11 individuals found to influence the CETP estimate. Exclusion

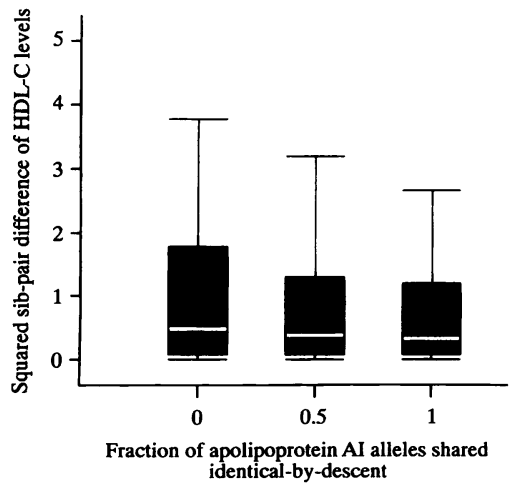

Figure 3. Distributions of squared sibling-pair differences of adjusted plasma HDL-C levels in siblings sharing none, one, or two apolipoprotein AI/CIII/AIV alleles identical-by-descent. Values corresponding to the boxes and whiskers are given in the legend to Fig. 2.

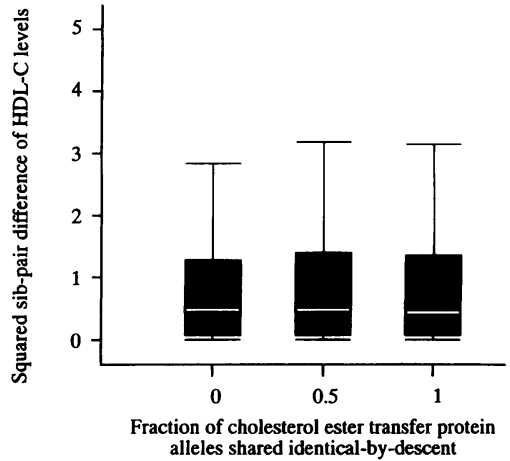

Figure 4. Distributions of squared sibling-pair differences of adjusted plasma HDL-C levels in siblings sharing none, one, or two cholesteryl ester transfer protein alleles identical-by-descent. Values corresponding to the boxes and whiskers are given in the legend to Fig. 2.

of the influential points did not significantly affect the variance estimates (Table III), indicating that these estimates are not an artifact of outliers.

The precision of the variance estimates was assessed using standard errors. Treating all sib-pair differences as uncorrelated observations we have determined standard error estimates of 18,18 , and $24 \%$ for hepatic lipase, apolipoprotein AI/CIII/ AIV, and CETP, respectively, using the delta method. As our data comprise nuclear families with sibship sizes of at least three, all individuals are represented in at least two pairs. Therefore the assumption of independent sib pairs made by Haseman and Elston (20) is not met and a degree of statistical efficiency in the error estimation is lost. Theoretically, more accurate error estimates can be determined using generalized least squares as discussed by Blackwelder and Elston (45), but generalized least squares analysis of our data (not shown), taking into account the correlations among sib pairs within sibships, indicated that the generalized least squares point estimates $\hat{h}_{g}^{2}$, and associated standard errors, are extremely sensitive to outliers and to the method of estimating the necessary covariance matrix. Given these considerations, the standard errors reported here provide only preliminary estimates of precision.

The inverse relationship between plasma HDL-C and plasma triglyceride levels was clearly evident in our sample. The effects of this association on the estimates of $\hat{h}_{g}^{2}$ were examined by analyzing regression adjusted HDL-C for the effects of age, sex, and plasma triglyceride levels. The systematic effects of plasma triglycerides did not significantly affect the proportion of variation in HDL-C levels that could be accounted for by allelic variation in hepatic lipase (19\%), apolipoprotein $\mathrm{AI} / \mathrm{CIII} / \mathrm{AIV}(24 \%)$, or CETP $(0 \%)$.

The combined effects of variation in hepatic lipase and apolipoprotein $\mathrm{AI} / \mathrm{CIII} / \mathrm{AIV}$ were examined by estimating the in-

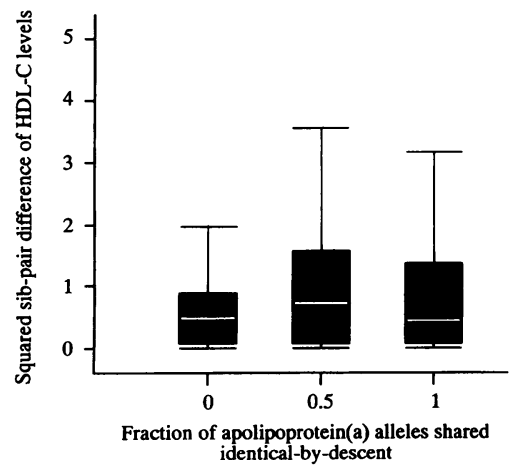

Figure 5. Distributions of squared sibling-pair differences of adjusted plasma HDL-C levels in siblings sharing none, one, or two apolipoprotein (a) alleles identicalby-descent. Values corresponding to the boxes and whiskers are given in the legend to Fig. 2. 


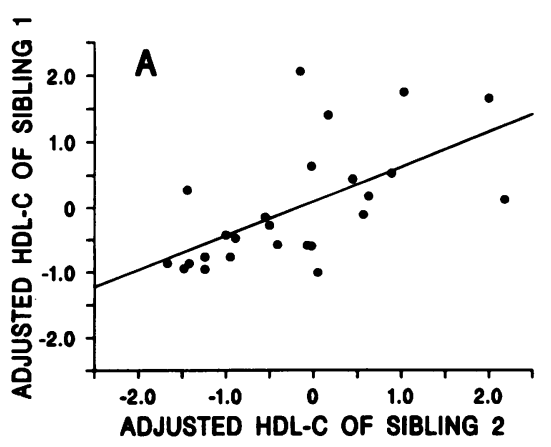

trapair correlation of HDL-C levels in sibling pairs sharing both hepatic lipase alleles and both apolipoprotein AI/CIII/ AIV alleles identical-by-descent, and in siblings pairs sharing no alleles at either locus (Fig. 6). A strong positive correlation ( $r=0.58, P=0.002$ ) was observed for siblings concordant for both alleles of both genes. No correlation $(r=-0.03, P$ $=0.9$ ) was observed for siblings sharing no alleles at either locus. The combined effects of variation at the hepatic lipase and apolipoprotein AI/CIII/AIV loci were also examined graphically. The distributions of squared sib-pair differences of adjusted HDL-C levels among siblings sharing both hepatic lipase and both apolipoprotein AI/CIII/AIV alleles identicalby-descent and among siblings sharing no alleles of these genes identical-by-descent were plotted (Fig. 7). The disparity in the distribution of squared sib-pair differences of HDL-C levels of siblings sharing no alleles versus siblings sharing identical alleles was clearly greater for hepatic lipase and apolipoprotein AI/CIII/AIV together than for either locus alone.

\section{Discussion}

According to current models of HDL metabolism, interindividual variation in plasma HDL-C levels is due to both environmental factors and allelic variation at several genetic loci. The number of genes involved and the fraction of the variance in HDL-C attributable to each is not known. In this study, we examined the effects of allelic variation in the genomic regions encoding hepatic lipase, apolipoprotein AI/CIII/AIV, and CETP on variation in the plasma HDL-C levels of normolipidemic individuals by comparing HDL-C levels of sibling pairs sharing different numbers of alleles identical-by-descent. Genetic variation accounted for a total of $56.5 \%$ of the variation in plasma HDL-C levels in this sample. This value is in good agreement with heritability indices for HDL-C levels derived from several family and twin studies $(12,13)$. The novel finding

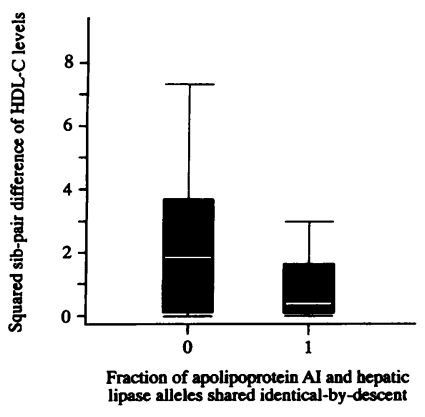

Figure 7. Distributions of squared sibling-pair differences of adjusted plasma HDL-C levels in siblings sharing no hepatic lipase and no apolipoprotein alleles, or both hepatic lipase and both apolipoprotein AI/CIII/ AIV alleles identical-by-descent. Values corresponding to the boxes and whiskers are given in the legend to Fig. 2. of our study is that a major fraction of the genetically determined variation in plasma HDL-C levels can be accounted for by allelic variation in hepatic lipase and the apolipoprotein AI/ CIII/AIV gene cluster.

Hepatic lipase is a lipolytic enzyme that hydrolyses lipoprotein triglyceride and phospholipid (46). A study of monozygotic twins suggested that hepatic lipase activity is strongly influenced by genetic factors (47), but the relationship between genetic variation in hepatic lipase and plasma HDL-C levels has not been determined previously. In the present study, allelic variation in the genomic region encoding hepatic lipase accounted for $25 \%$ of the variance in plasma HDL-C levels. Adjustment for plasma triglyceride levels did not significantly affect this estimate, indicating that the association is not secondary to hepatic lipase-mediated variation in plasma triglyceride levels. The association between plasma HDL-C levels and allelic variation in the genomic region encoding hepatic lipase suggests that variation in the hepatic lipase gene causes interindividual variation in hepatic lipase activity, and consequently interindividual variation in plasma HDL-C levels. This hypothesis is consistent with the strong negative correlation between plasma HDL-C levels and hepatic lipase activity that has been reported in several studies $(26,48,49)$.

Since hepatic lipase activity was not measured in this study, we can only speculate about the physiologic mechanisms underlying the association observed between plasma HDL-C levels and allelic variation in hepatic lipase. The association was not due to outliers, therefore functionally distinct hepatic lipase alleles must be common in the population. The possible phenotypic consequences of alleles that confer low hepatic lipase activity should be most evident in families segregating mutations that abolish hepatic lipase activity entirely, since these mutations can be considered an extreme form of allelic variation. Unfortunately, in the few kindreds that have been described in detail, interpretation of the effects of the hepatic lipase mutation on plasma HDL-C levels is confounded by the presence of other monogenic disorders of lipoprotein metabolism (50), or by the fact that hepatic lipase activities range from almost zero to near normal levels in individuals who are obligate heterozygotes for a mutant hepatic lipase allele (51). Thus the effect on HDL-C levels of hepatic lipase alleles that confer low hepatic lipase activity is unclear.

Alleles that confer increased hepatic lipase activity may also be present in the population. Although such alleles have not been reported, Blades et al. (52) found that mean postheparin plasma hepatic lipase activity was $25 \%$ higher in men who had low HDL-C levels in the absence of hypertriglyceridemia than in men with normal HDL-C. Therefore alleles that confer high 
hepatic lipase activity may be systematically associated with low plasma HDL-C levels.

The genes encoding apolipoproteins AI, CIII, and AIV are located in a cluster spanning $\sim 20 \mathrm{~kb}$ on the long arm of chromosome 11 (53). Several studies have investigated the relationship between polymorphisms in the apolipoprotein AI/CIII/ AIV gene cluster and plasma HDL-C levels $(18,19)$, but the cumulative effect of allelic variation in these genes on HDL-C levels in the population has not been determined. In this study, allelic variation in the genomic region containing the apolipoprotein $\mathrm{AI} / \mathrm{CIII} / \mathrm{AIV}$ gene cluster accounted for $22 \%$ of the variance in plasma HDL-C levels. Since these genes are so closely linked, it was not possible to determine their individual contributions to the inter-individual variation in HDL-C levels.

Apolipoprotein $\mathrm{AI}$ is the major protein component of $\mathrm{HDL}$, and plasma levels of apolipoprotein AI and HDL-C are strongly correlated (27). The association between HDL-C levels and allelic variation in the genomic region encoding apolipoprotein $\mathrm{AI} / \mathrm{CIII} / \mathrm{AIV}$ may be due to common, functionally distinct apolipoprotein $\mathrm{AI}$ alleles that are associated with different plasma levels of HDL-C. Alleles bearing point mutations that abolish apolipoprotein AI gene expression are associated with low HDL-C levels $(24,54)$. Individuals homozygous for such mutations have little or no plasma HDL-C, while heterozygotes have HDL-C levels that are approximately half those of their unaffected siblings. The observation that a $50 \%$ decrease in apolipoprotein AI gene function (corresponding to the loss of one allele) results in a marked decrease in HDL-C suggests that alleles that confer a moderate decrease in apolipoprotein $\mathrm{AI}$ expression will be associated with a moderate decrease in HDLC levels.

The physiological functions of apolipoprotein CIII and apolipoprotein AIV are not fully understood, and the effects of allelic variation in these genes on plasma HDL-C levels is difficult to predict. Association studies have indicated a relationship between apolipoprotein CIII polymorphisms and plasma triglyceride levels $(55,56)$, and it is possible that the effects of allelic variation in this genomic region observed in the present study are secondary to variation in plasma triglycerides levels. The confounding effects of triglyceride levels on variation in HDL$\mathrm{C}$ were accommodated in two ways. First, we excluded a priori 13 offspring with plasma triglyceride levels $>200 \mathrm{mg} / \mathrm{dl}$, thereby limiting the study to normotriglyceridemic individuals. Second, we adjusted the HDL-C values of the remaining offspring for the effects of triglyceride concentration. If variation in HDL-C that is apparently attributable to a candidate locus is actually secondary to variation in plasma triglyceride levels, then this adjustment should decrease the proportion of the variance in HDL-C that is attributable to the candidate gene. Adjustment of HDL-C for plasma triglyceride levels did not affect the estimates of variation in HDL-C due to apolipoprotein AI/CIII/ AIV. Therefore the effect of variation in these genes on HDL$\mathrm{C}$ levels is not secondary to variation in plasma triglyceride levels. It is also possible that allelic variation in apolipoprotein CIII may have effects on plasma HDL-C levels that are independent of plasma triglyceride concentration. von Eckardstein et al. (57) described a mutation in the apolipoprotein CIII gene that is associated with very low levels of apolipoprotein CIII ( $\sim 50 \%$ of normal), very high HDL-C ( $>99$ percentile), and unremarkable triglyceride levels in two heterozygous women (a mother and her daughter). Apolipoprotein CIII alleles that confer moderately decreased plasma levels of apolipoprotein
CIII may therefore increase HDL-C levels in normotriglyceridemic individuals in the general population.

The CETP catalyzes the exchange of cholesteryl esters and triglycerides among lipoproteins (58). A major role for CETP in HDL metabolism is indicated by the markedly elevated plasma HDL-C levels in individuals with CETP deficiency (25), but measurements of plasma CETP activity show little or no correlation with plasma HDL-C levels in normolipidemic individuals $(59,60)$. In this study, variation in the CETP gene did not account for any of the variance in plasma HDL-C levels. Therefore allelic variation in the gene encoding CETP does not appear to be a significant cause of variation in HDL-C levels in our sample.

To determine whether genes unrelated to HDL-C metabolism would show spurious correlation with plasma HDL-C levels in this analysis, we examined the effects of polymorphism in the apolipoprotein(a) gene on variation in plasma HDL-C levels. Apolipoprotein (a) appears to be metabolically unrelated to plasma HDL, therefore allelic variation in the apolipoprotein(a) gene should not influence plasma HDL-C levels. In this study all four parental apolipoprotein(a) alleles were distinguished in $\mathbf{4 8}$ families. The Haseman-Elston regression procedure indicated that none of the variation in plasma HDL-C levels was attributable to polymorphism in the apolipoprotein (a) gene. The analysis of apolipoprotein(a) serves as a negative control in this study, suggesting that the results obtained for the candidate genes are not simply the fortuitous result of sampling error or statistical artifact.

An important question that remains to be answered is the extent of the interaction between hepatic lipase and apolipoprotein AI/CIII/AIV. Current analytical methods do not provide simultaneous estimates of the phenotypic effect of variation at several loci, therefore the cumulative effects of allelic variation in hepatic lipase and apolipoprotein AI/CIII/AIV could not be directly determined. We have examined this question qualitatively by plotting the squared sib-pair differences of HDL-C levels among sibs sharing both hepatic lipase and both apolipoprotein AI/CIII/AIV alleles identical by descent (Fig. 7). The disparity in the distributions of squared sib-pair differences of HDL-C levels among sibs sharing different proportions of alleles identical by descent was clearly greater for hepatic lipase and apolipoprotein AI/CIII/AIV considered together than for either locus alone. This observation suggests that the variance in HDL-C levels attributable to combined effects of these two loci is greater than the fraction contributed by either locus alone.

The biological interpretation of quantitative genetic data may be strongly influenced by the type of statistical analysis applied (61). In this study we used the sibling-pair method of Haseman and Elston (20) because the procedure is based on simple genetic and statistical assumptions. Additional analyses were performed to exclude the possibility that our results are an artifact of the adjustment procedure, the presence of outliers, or covariates such as age, sex, and plasma triglyceride levels. In addition, the results of the Haseman-Elston analysis were corroborated using the following reasoning: if allelic variation in the genomic regions encoding hepatic lipase and apolipoprotein AI/CIII/AIV accounts for the major fraction of the genetically determined variation in plasma HDL-C levels, then HDL$C$ levels should be strongly correlated in siblings sharing two identical alleles at both of these loci. The correlation between siblings with different alleles of both genes should be much weaker. Alternatively, if the heritable component of plasma 
HDL-C levels is due primarily to polygenic and cultural factors then intra-pair correlations should be similar irrespective of the inheritance of hepatic lipase and apolipoprotein AI/CIII/AIV alleles. In the present data set, plasma HDL-C levels were strongly correlated $(r=0.58, P=0.002)$ between siblings sharing both alleles of hepatic lipase and apolipoprotein AI/ CIII/AIV identical by descent, but not between siblings sharing no alleles of either gene $(r=-0.03, P=0.9)$. Taken together, these correlations indicate that allelic variation in hepatic lipase and apolipoprotein AI/CIII/AIV account for a major fraction of the genetically determined variation in HDL-C levels, and that factors such as polygenes and shared environment are much less important.

In summary, we have examined the genetic determinants of plasma HDL-C levels in normolipidemic humans. Our data indicate that $\sim 50 \%$ of the variation in HDL-C levels can be accounted for by genetic factors. A major fraction of the genetically determined variation is conferred by allelic variation in the genomic regions encoding hepatic lipase and apolipoprotein $\mathrm{AI} / \mathrm{CIII} / \mathrm{AIV}$. Interindividual variation in traits such as HDL$\mathrm{C}$, which are clearly influenced by genetic factors but are continuous and approximately normally distributed in the population, have traditionally been considered to reflect slight functional variations in numerous genes. The results of this study indicate that genetic variation in HDL-C levels is determined largely by variation in two genomic regions. These findings suggest that interindividual variation in some traits traditionally considered to be polygenic may be due primarily to functional variation in a few key genes, rather than to the aggregate effect of subtle variations in a large number of genes.

\section{Acknowledgments}

We thank Kathy Schutt for excellent technical assistance, Richard Verstraete and Carla Leffert for help in recruiting families, Robert I. Barnes from the McDermot Center for help with identification of appropriate microsatellites, and Tommy Hyatt and Kathy Schueler from Dr. Helen Hobbs' lab for apolipoprotein(a) genotyping. We wish to express our sincere thanks to Dr. Helen Hobbs for her enthusiastic support, and to Dr. Chris Amos for critical review of the manuscript.

This project was supported by a grant from the American Heart Association, Texas Affiliate, by National Institutes of Health grants HL29252 and MO1-RR-00633, by the Southwestern Medical Foundation, The Moss Heart Foundation of Dallas, and by an unrestricted grant from Bristol-Myers-Squibb.

\section{References}

1. National Cholesterol Education Program. Second report of the expert panel on detection, evaluation, and treatment of high blood cholesterol in adults.

2. Miller, G. J., and N. E. Miller. 1975. Plasma high-density-lipoprotein concentration and development of ischaemic heart disease. Lancet. i:16-19.

3. Gordon, T., W. P. Castelli, M. C. Hjortland, W. B. Kannel, and T. R. Dawber. 1977. High density lipoproteins as a protective factor against coronary heart disease: the Framingham study. Am. J. Med. 62:707-714.

4. Wiklund, O., L. Wilhelmsen, D. Elmfeldt, H. Wedel, J. Valek, and A. Gustafson. 1980. Alpha-lipoprotein cholesterol concentration in relation to subsequent myocardial infarction in hypercholesterolemic men. Atherosclerosis. 37:4753.

5. Frick, M. H., M. O. Elo, and K. Haapa. 1987. Helsinki Heart Study: primary prevention trial with gemfibrozil in middle-aged men with dyslipidemia. Safety of treatment, changes in risk factors, and incidence of coronary heart disease. $N$. Engl. J. Med. 317:1237-1245.

6. Gordon, D. J., J. L. Probstfeld, and R. J. Garrison. 1989. High density lipoprotein cholesterol and cardiovascular disease: four prospective American studies. Circulation. 79:8-15.

7. Berchtold, P., M. Berger, V. Jorgens, C. Dweke, E. Chantelau, F. A. Gries, and H. Zimmerman. 1981. Cardiovascular risk factors and HDL-cholesterol levels in obesity. Int. J. Obes. 5:1-10.

8. Sutherland, W. H., W. A. Temple, E. R. Nye, and G. P. Herbison. 1980. Adiposity, lipids, alcohol consumptions, smoking, and gender. Am. J. Clin. Nutr. 33:2581-2587.

9. Schieken, R. M. 1991. Effect of exercise on lipids. Ann. N. Y. Acad. Sci. 623:269-274.

10. Craig, W. Y., G. E. Palomaki, and J. E. Haddow. 1989. Cigarette smoking and serum lipid and lipoprotein concentrations; an analysis of published data. $\mathrm{Br}$. Med. J. 298:784-788.

11. Wannamethee, G., and A. G. Shaper. 1992. Blood lipids: the relationship with alcohol intake, smoking, and body weight. J. Epidemiol. Community Health. 46:197-202.

12. Heller, D. A., U. De Faire, N. L. Pedersen, G. Dahlen, and G. E. McClearn. 1993. Genetic and environmental influences on serum lipid levels. N. Engl. J. Med. 328:1150-1156.

13. Steinmetz, J., E. Boerwinkle, R. Gueguen, S. Visvikis, J. Henny, and G. Siest. 1992. Multivariate genetic analysis of high density lipoprotein particles. Atherosclerosis. 92:219-227.

14. MacCluer, J. W. 1992. Biometrical studies to detect new genes with major effects on quantitative risk factors for atherosclerosis. Curr. Opin. Lipidol. 3:114121.

15. Friedlander, Y., J. D. Kark, and Y. Stein. 1986. Complex segregation analysis of low levels of plasma high-density lipoprotein cholesterol in a sample of nuclear families in Jerusalem. Genet. Epidemiol. 3:285-297.

16. Moll, P. P., C. F. Sing, R. R. Williams, S. J. T. Mao, and B. A. Kottke. 1986. The genetic determination of apolipoprotein A-I levels measured by radioimmunoassay. A study of high risk pedigrees. Am. J. Hum. Genet. 38:361-372.

17. Ferrell, R. E. 1992. Genetics of the apolipoproteins and the contribution of allelic variation to quantitative variation in lipid and lipoprotein levels in the population. Curr. Opin. Lipidol. 3:122-127.

18. Lusis, A. J. 1988. Genetic factors affecting blood lipoproteins: the candidate gene approach. J. Lipid Res. 29:397-429.

19. Humphries, S. E. 1988. DNA polymorphisms of the apolipoprotein genes: their use in the investigation of the genetic component of hyperlipidaemia and atherosclerosis. Atherosclerosis. 72:89-108.

20. Haseman, J. K., and R. C. Elston. 1972. The investigation of linkage between a quantitative trait and a marker locus. Behav. Genet. 2:2-19.

21. Boerwinkle, E., C. C. Leffert, J. Lin, C. Lackner, G. Chiesa, and H. H. Hobbs. 1992. Apolipoprotein (a) gene accounts for greater than $90 \%$ of the variation in plasma lipoprotein (a) concentrations. J. Clin. Invest. 90:52-60.

22. Amos, C. I., R. C. Elston, S. R. Srinivasan, A. F. Wilson, J. L. Cresanta, L. J. Ward, and G. S. Berenson. 1987. Linkage and segregation analyses of apolipoproteins $\mathrm{A} 1$ and $\mathrm{B}$, and lipoprotein cholesterol levels in a large pedigree with excess coronary heart disease: the Bogalusa Heart Study. Genet. Epidemiol. 4:115-128.

23. Connelly, P. W., G. F. Maguire, M. Lee, and J. A. Little. Plasma lipoproteins in familial hepatic lipase deficiency. 1990. Arteriosclerosis. 10:40-48.

24. Deeb, S. S., M. C. Cheung, R. Peng, A. Wolf, R. Stern, J. J. Albers, and R. H. Knopf. A mutation in the apolipoprotein A-I gene: dominant effect on the level and characteristics of plasma high density lipoproteins. 1991. J. Biol. Chem. 266:13654-13660.

25. Inazu, A., M. I. Brown, C. B. Hesler, L. B. Agellon, J. Koizumi, K. Takata, Y. Maruhama, H. Mabuchi, and A. R. Tall. 1990. Increased high-density lipoprotein levels caused by a common cholesteryl-ester transfer protein gene mutation. N. Engl. J. Med. 323:1234-1238.

26. Applebaum-Bowden, D., S. M. Haffner, P. W. Wahl, J. J. Hoover, G. R. Warnick, J. J. Albers, and W. R. Hazzard. 1987. Postheparin plasma triglyceride lipases. Relationships with very low density lipoprotein triglyceride and high density lipoprotein cholesterol. Arteriosclerosis. 5:273-282.

27. Gylling, H., G. L. Vega, and S. M. Grundy. 1992. Physiologic mechanisms for reduced apolipoprotein A-I concentrations associated with low levels of high density lipoprotein cholesterol in patients with normal plasma lipids. J. Lipid Res. 33:1527-1539.

28. Warnick, G. R., M. C. Cheung, and J. J. Albers. 1983. Comparison of current methods for high-density lipoprotein cholesterol quantitation. Clin. Chem. 29:538-542.

29. Reina, M., and S. Deeb. 1992. SSCP polymorphism in the human hepatic triglyceride lipase (LIPC) gene. Hum. Mol. Genet. 1:453.

30. Bhattacharya, S., D. Ameis, P. Cullen, T. M. Narcisi, J. Bayliss, H. Greten, M. C. Schotz, and J. Scott. 1991. VNTR polymorphism in the hepatic lipase (LIPC) gene. Nucleic Acids Res. 19:5088.

31. Weissenbach, J., G. Gyapay, C. Dib, A. Vignal, J. Morissette, P. Millasseau, G. Vaysseux, and M. Lathrop. 1992. A second generation linkage map of the human genome. Nature (Lond.). 359:794-801.

32. Hudson, T. J., M. Engelstein, M. K. Lee, E. C. Ho, M. J. Rubenfield, C. P. Adams, D. E. Housman, and N. C. Dracopoli. 1992. Isolation and chromosomal assignment of 100 highly informative human simple sequence repeat polymorphisms. Genomics. 13:622-629.

33. Beckman, J. S., J. Tomfohrde, R. I. Barnes, M. Williams, O. Broux, I. 
Richard, J. Weissenbach, and A. M. Bowcock. 1993. A linkage map of human chromosome 15 with an average resolution of $2 \mathrm{cM}$ and containing 55 polymorphic microsatellites. Hum. Mol. Genet. 2:2019-2030.

34. Xu, C.-F., F. Angelico, M. Ben, and S. Humphries. 1993. Role of genetic variation at the apo AI-CIII-AIV cluster in determining plasma apo AI levels in boys and girls. Genet. Epidemiol. 10:113-122.

35. Luo, X. Y., G. A. Evans, and M. Litt. 1990. Dinucleotide repeat polymorphism at the D11S490 locus. Nucleic Acids Res. 18:7470.

36. Zuliani, G., and H. H. Hobbs. 1990. Tetranucleotide repeat polymorphism in the apolipoprotein C-III gene. Nucleic Acids Res. 18:4299.

37. Weber, J. L., A. E. Kwitek, and P. E. May. 1990. Dinucleotide repeat polymorphisms at the D11S419 and CD3D loci. Nucleic Acids Res. 18:4036.

38. Shen, Y., A. T. Thompson, K. Holman, D. F. Callen, G. R. Sutherland, and R. I. Richards. 1992. Four dinucleotide repeat polymorphisms on human chromosome 16 at D16S289, D16S318, D16S319 and D16S320. Hum. Mol. Genet. 1:773.

39. Läckner, C., J. C. Cohen, and H. H. Hobbs. 1993. Molecular definition of the extreme size polymorphism in apolipoprotein(a). Hum. Mol. Genet. 2:933940.

40. Cohen, J. C., G. Chiesa, and H. H. Hobbs. 1993. Sequence polymorphisms in the apolipoprotein(a) gene. Evidence for dissociation between apolipoprotein (a) size and plasma lipoprotein(a) levels. J. Clin. Invest. 91:1630-1636.

41. Heiss, G., N. J. Johnson, S. Reiland, C. E. David, and H. A. Tyroler 1980. The epidemiology of plasma high density lipoprotein cholesterol levels. The Lipid Research Clinics Program Prevalence Study. Summary. Circulation. 62:116-136.

42. Falconer, D. S. 1989. Introduction to Quantitative Genetics. 3rd ed. Longman Group UK Limited, Harlow, UK. 148-186.

43. Bishop, Y. M. M., S. E. Fienberg, and P. W. Holland. 1975. Discrete Multivariate Analysis: Theory and Practice. MIT Press, Cambridge, Mass. 486502.

44. Belsley, D. A., E. Kuh, and R. E. Welsch. 1980. Regression Diagnostics. John Wiley and Sons Ltd., New York. 11-14.

45. Blackwelder, W. C., and R. C. Elston. 1982. Power and robustness of the sib-pair linkage tests and extension to larger sibships. Commun. Statist. Theor. Meth. 11:449-4.

46. Grosser, J., O. Schrecker, and H. Greten. 1981. Function of hepatic triglyceride lipase in lipoprotein metabolism. J. Lipid Res. 22:437-442.

47. Kuusi, T., Y. A. Kesaniemi, M. Vueristo, T. A. Miettinen, and M. Koskenvuo. 1987. Inheritance of high density lipoprotein and lipoprotein lipase and hepatic lipase activity. Arteriosclerosis. 7:421-425.

48. Kuusi, T., P. Saarinen, and E. A. Nikkila. 1980. Evidence for the role of hepatic lipase in the metabolism of plasma high density lipoproteins in man. Atherosclerosis. 36:589-593.
49. Jackson, R. L., M. T. Yates, C. A. McNerney, and M. L. Kashyap. 1990 Relationship between post-heparin plasma lipases, triglycerides, and high density lipoproteins in normal subjects. Horm. Metab. Res. (Suppl.) 22:289-294.

50. Auwerx, J. H., S. P. Babirak, J. E. Hokanson, G. Stahnke, H. Will, S. S. Deeb, and J. D. Brunzell. 1990. Coexistence of abnormalities of hepatic lipase and lipoprotein lipase in a large family. Am. J. Hum. Genet. 46:470-477.

51. Hegele, R. A., J. A. Little, C. Vezina, G. F. Maguire, L. Tu, T. S. Wolever, D. J. A. Jenkins, and P. W. Connelly. 1993. Hepatic lipase deficiency. Clinical, biochemical, and molecular genetic characteristics. Arteriosclerosis Thromb. 13:720-728.

52. Blades, B., G. L. Vega, and S. M. Grundy. 1993. Activities of lipoprotein lipase and hepatic lipase in postheparin plasma of patients with low concentrations of HDL cholesterol. Arteriosclerosis Thromb. 13:1227-1235.

53. Karathanasis, S. K. 1986. Apolipoprotein multigene family: tandem organization of the apolipoprotein AIV, AI and CIII genes. Proc. Natl. Acad. Sci. USA 82:6374-6378.

54. Ng, D. S., L. A. Leiter, C. Vezina, P. W. Connelly, and R. A. Hegele. 1994. Apolipoprotein AI Q [-2]X causing isolated apolipoprotein A-I deficiency in a family with analphalipoproteinemia. J. Clin. Invest. 93:223-229.

55. Dammerman, M., L. A. Sandkuijl, J. L. Halaas, W. Chung, and J. L. Breslow. 1993. An apolipoprotein CIII haplotype protective against hypertriglyceridemia is specified by promoter and $3^{\prime}$ untranslated region polymorphisms. Proc. Natl. Acad. Sci. USA. 90:4562-4566.

56. Ordovas, J. M., F. Civeira, J. Genest, S. Craig, A. H. Robbins, T. Meade, M. Pocovi, P. M. Frossard, U. Maharani, P. W. F. Wilson, et al. 1991. Restriction fragment length polymorphism of the apolipoprotein A-I, C-III, A-IV gene locus: relationships with lipids, apolipoproteins, and premature coronary artery disease. Atherosclerosis. 87:75-86.

57. von Eckardstein, A., H. Holz, M. Sandkamp, W. Weng, H. Funke, and G. Assman. 1991. Apolipoprotein C-III( Lys $_{58} \rightarrow$ Glu $)$. Identification of an apolipoprotein C-III variant in a family with hyperalphalipoproteinemia. J. Clin. Invest. 87:1724-1731.

58. Tall, A. R. 1993. Plasma cholesteryl ester transfer protein. J. Lipid Res 34:1255-1274

59. Freeman, D. J., B. A. Griffin, E. Murray, G. M. Lindsay, D. Gaffney, C. J. Packard, and J. Shepherd. 1993. Smoking and plasma lipoproteins in man: effects on low density lipoprotein cholesterol levels and high density lipoprotein subfraction distribution. Eur. J. Clin. Invest. 23:630-640.

60. Marcel, Y. L., R. McPherson, M. Hogue, H. Czarnecka, Z. Zawadzki, P. K. Weech, M. E. Whitlock, A. R. Tall, and R. W. Milne. 1990. Distribution and concentration of cholesteryl ester transfer protein in plasma of normolipidemic subjects. J. Clin. Invest. 85:10-17.

61. Mitchell-Olds, T., and J. Bergelson, 1990. Statistical genetics of an annual plant, Impatiens capensis. I. Genetic basis of quantitative variation. Genetics. 124:407-415. 